\title{
Una aproximación teórico crítica al análisis de la práctica educativa sobre educación moral
}

Concepción Borrego de Dios

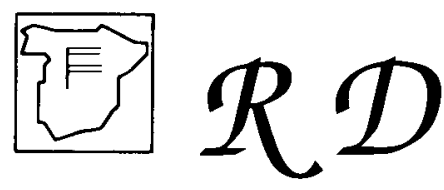

La directividad de la conducta y su definición moral implica algún tipo de representación para guiarla. Neutra y lógico-formal para unos (PiagetKoblberg), basada en el pensamiento; social, cultural y narrativa, $y$ coloreada por tanto de sentimientos, para otros (Bruner, Bozbovich). En este artículo se bace un acercamiento teórico y práctico a estos modelos $y$ al de $B$. Benstein a través de un diseño práctico de educación moral en primaria.

\section{INTRODUCCION Y PERSPECTIVAS TEORICAS}

La Reforma Educativa ha dado lugar a diferentes alternativas en torno a la forma de conseguir los objetivos encaminados a la educación socio-personal y moral de los niños que difieren en el grado de transversalidad o de especificidad que defienden. Al mismo tiempo que la posición oficial del Ministerio de Educación se ha inclinado hacia un planteamiento transversal, han surgido también propuestas, que aceptando básicamente la idea del tratamiento transversal, son favorables a un tratamiento específico de estas cuestiones como un medio de garantizar su tratamiento transversal (Martinez y Puig (Coord.) 1991; Borrego de Dios (Ed.) 1992a). Una idea favorable al tratamiento específico de la educación socio-personal y moral nos condujo a diseñar un ámbito de experiencias de «Educación para la vida en sociedad» en el Diseño Curricular de la Comunidad Autónoma Andaluza (Diseños Curriculares. Junta de Andalucía. 1990, pp. 409-469) y a la posterior inclusión de sus contenidos en el Area de conocimiento del medio socio-natural en la Educación Primaria (Decreto de Educación Primaria. Junta de Andalucía 1992). La entrada en vigor de la Reforma Educativa en el curso 1992-93 en ese nivel hizo posible la elaboración del proyecto Aprender a vivir juntos en la escuela (Borrego de Dios (Coord.) (1992b), estructurado para facilitar la puesta en práctica de la educación socio-personal y moral en las aulas. El proyecto, inspirado en un planteamiento de investigaciónacción (Carr y Kemmis, 1986; Elliot 1990), está concebido como una propuesta práctica abierta a la experimentación de los profesores quienes deben comprobar críticamente su validez como instrumento de educación socio-personal en las aulas. Aprender a vivir juntos en la escuela se estructura en tres bloques temáticos que abor- 
dan respectivamente el conocimiento de las personas, las relaciones interpersonales y la vida en grupo. Cada uno de ellos incluye diferentes módulos que desarrollan más pormenorizadamente, a través de diferentes tipos de actividades aspectos parciales su propia problemática.

Este artículo se propone realizar una reflexión crítica en torno a algunas de las actividades contenidas en el propio Proyecto y a su puesta en práctica, a partir de las primeras observaciones llevadas a cabo mediante una metodología etnográfica en aulas de educación primaria. Realizaremos esa reflexión enfocando dos tipos de cuestiones. La primera de ellas gira en torno a algunos de los problemas que implica el desarrollo de un diseño curricular en la práctica del aula. Enfocaremos esta cuestión desde los recientes planteamientos de Basil Bernstein (1990a y 1990b) acerca del discurso pedagógico como un proceso de recontextualización de distintos tipos de conocimiento en la práctica educativa. Se deriva de esta posición que el curriculum diseñado es filtrado a través de la ideología de los profesores y convertido en prácticas pedagógicas concretas en las aulas (Ingillery en prensa), de manera que es posible distinguir entre distintos niveles de concreción del curriculum: el oficial, es decir, el establecido por los estamentos gubernamentales que prescriben el curriculum básico, el posible que se refiere a la transformación del curriculum oficial que realizan los profesores en atención a lo que estiman ser las condiciones de sus alumnos y el curriculum enseñado que se refiere a lo que el profesor transmite realmente a sus alumnos y que se desprende de la observación de lo que los profesores hacen o dicen en sus clases (Espínola, en prensa).

El discurso pedagógico integra y relaciona dos discursos: un discurso instruccional que crea competencias o habilidades especializadas que deben ser adquiridas y un discurso regulativo que crea orden social y se expresa a través del control y la disciplina en el aula. En palabras del propio Bernstein:

«El discurso instruccional se ocupa de la transmisiónladquisicion de competencias especificas y el discurso regulativo se dedica a la transmisión de principios de orden, relación e identidad» (1990a. Traduc. española. 1993. pág 216).

El discurso pedagógico implica la inserción de uno en otro, de manera que se funden en un único discurso pedagógico en el que el discurso instruccional se subordina al discurso dominante, el discurso regulativo. Mientras que el discurso instruccional opera a través de reglas que establecen la secuencia y el ritmo de los aprendizajes, así como el criterio que determina lo que es legítimo y lo que no lo es en el proceso educativo, el discurso regulativo lo hace a través de reglas jerárquicas que determinan el orden social y el carácter, que se plantean como la condición para que se produzca la conducta apropiada en la relación pedagógica. Estas reglas que determinan el discurso pedagógico pueden ser más o menos explícitas. Cuando son explícitas, las relaciones de autoridad y la conducta que se pide al niño son claras y las relaciones de poder están explícitamente definidas, mientras que cuando son implícitas, las relaciones entre el transmisor y el adquiriente están enmascaradas o escondidas. (Sadovnik, 1991).

Conviene señalar que, en el caso que nos ocupa, la educación socio-personal y moral pretende transmitir como contenidos instruccionales explícitos habilidades que hacen al orden moral y a las reglas de funcionamiento social que están implícitos en el caso de la transmisión de otros tipos de habilidades y conocimientos escolares no morales. Desde la perspectiva de Bernstein, el tratamiento específico de la educación socio-personal y moral transformaría en contenido instruccional explícito el propio discurso regulativo. Puesto que el orden moral permanece usualmente oculto en la práctica educativa, la incorporación de la educación socio-personal y moral en el currículum escolar convierte en explícitos contenidos educativos que están siem- 
pre presentes en la enseñanza de cualquier área curricular aunque usualmente al nivel del currículum oculto (Borrego de Dios, 1992b).

La segunda cuestión se centra en dos tipos de planteamientos, diferentes y posiblemente complementarios (Benhabid, 1992), en torno a la educación moral: la tradición cognitivo-evolutiva que arranca de los planteamientos iniciales de Piaget (1932) y que tiene su máxima expresión en los planteamientos educativos kohlberianos (1969, 1971, 1976), y las implicaciones educativas en el terreno de la educación moral de los recientes enfoques que parten de una consideración interpretativista del mundo social de los que se desprende una posición narrativa del ámbito socio-personal y moral (Bruner 1988, 1990; Gilligan 1982).

La tradición cognitivo-evolutiva ha concebido el desarrollo moral como un despliegue interno de estadios en los que un tipo de moralidad imperfecta (heteronomía moral o convencionalidad) era sucedido por un tipo de moralidad autónoma e independiente de las normas socialmente establecidas (autonomía moral o nivel postconvencional). Mientras que Piaget hacía depender el desarrollo moral de la superación de una característica central del pensamiento infantil, el egocentrismo, Kohlberg concebía el desarrollo moral como dependiente de una única estructura cognitiva central en la que el desarrollo de los juicios morales corre parejo al desarrollo de los conceptos lógicos y físicos, si bien, matizaba que, el desarrollo de éste último tipo de conceptos, siendo necesario, no era suficiente para el desarrollo de los correspondientes niveles de desarrollo moral. El planteamiento estructural de Kohlberg sobre el desarrollo moral se traduce en un planteamiento de la educación moral que aspira a la promoción de estructuras de juicio basadas en principios universales susceptibles de ser aplicables a toda la humanidad. Se trata de principios de justicia tales como la libertad, igualdad y reciprocidad, principios que cualquier persona defendería si ignorara su posición social o si fuera el menos privilegiado dentro de una determinada sociedad:

«Los principios escogidos desde este punto de vista son, en primer lugar, la máxima libertad personal compatible con la idéntica libertad de los otros, y, en segundo lugar, la no desigualdad de bienes que deben ser para el beneficio de todos, incluyendo a los menos favorecidos» (Kohlberg 1975, pág. 97).

Dado que el objetivo de la educación moral consiste en el desarrollo de principios de justicia, Kohlberg afirmaba en 1975 que «el enfoque evolutivo moral restringe la educación de los valores a aquello que es moral, o más específicamente a la justicia»( 1975, pág. 101), declarando que quedaban fuera de los objetivos de la educación moral el mundo de los valores personales, políticos y religiosos debido a la dificultad de encontrar principios universales y dirección del desarrollo en estos campos. Entendida la educación moral como centrada en la justicia y diferenciada de la educación de valores o de la educación de las vertientes afectivas de la personalidad, la educación moral se identifica con la educación cívica, dando por supuesto que se trata de una educación en principios filosóficos universales antes que en las exigencias de una sociedad particular.

Una interpretación diferente acerca de la educación socio-personal y moral se deriva de posiciones interpretativistas acerca del mundo social como la defendida por Bruner $(1986,1990)$ en sus últimos libros, en los que plantea la existencia de dos modalidades de pensamiento, la modalidad paradigmática de la lógica y la ciencia que se corresponde con el conocimiento de la naturaleza y la modalidad del relato y la narrativa que se centra en torno al drama de las intenciones humanas y sus visicitudes (J. Bruner 1988). Mientras que la ciencia hace abstracción de lo particular en busca de niveles de universalidad progresivamente mayores y trata de construir un mundo invariable a pesar de las intenciones y de los conflictos humanos, las narrativas se refieren a sucesos anclados en el espacio y en el tiempo y logran la universalidad por su sensibilidad al contexto. Las narrativas en sus diferentes formas 
constituyen medios a través de los que una sociedad expresa sus creencias compartidas que propone como modelos a las generaciones jóvenes al mismo tiempo que como mecanismos de distanciamiento de esos mismos supuestos culturalmente compartidos de manera que sea posible su reelaboración y recreación colectiva. De esta forma, la narrativa, al mismo tiempo que expresa las acciones que son permisibles en una cultura y moldea a los individuos para desempeñar papeles activos y sentir emociones compatibles con ella, permite la negociación, la recreación de los valores sociales y la creación de nuevos mundos posibles.

Carol Gilligan descubre también una sensibilidad contextual y narrativa en la forma en que las mujeres describen su posición ante problemas morales en su libro In a different voice (1982) en el que nos habla de "dos modos de hablar de problemas morales, dos modos de describir la relación entre el otro y yo» que ella asocia a dos voces diferentes, la masculina y la femenina que representan diferentes modos de pensamiento incluso dentro de cada sexo. Ambas posiciones ante la moralidad y las relaciones interpersonales conceden diferentes grados de importancia a la separación del yo individual o a la conexión con los otros, a la vida autónoma alrededor del trabajo o hacia las relaciones de interdependencia en relación con el amor y el cuidado. A través de entrevistas con mujeres en etapas críticas de sus vidas, Gilligan revela un tipo de moralidad basada en la conexión y en la trama de relaciones interpersonales que se rige por pautas diferentes a la moralidad descrita por Kohlberg que hace hincapié en la consideración del individuo y en un entendimiento reflexivo de los derechos humanos:

«Si empezamos el estudio de las mujeres y derivamos de sus vidas las elaboraciones del desarrollo, empiezan a surgir los lineamientos de una concepción moral distinta de la descrita por Freud, Piaget o Koblberg y conforman una descripción diferente del desarrollo. En esta concepción, el problema moral surge de responsabilidades en conflicto y no de derechos competitivos, y para su resolución pide un modo de pensar que sea contextual y narrativo en lugar de formal y abstracto» (Gilligan (1982). Traduc. española. 1985, pág. 42).

En los siguientes apartados analizaremos estas cuestiones a partir de tres actividades educativas contenidas en el proyecto Aprender a vivir juntos en la escuela. En la primera de ellas los niños deben realizar una representación de sí mismos a través de un dibujo con el objetivo el favorecer el conocimiento de los niños que conviven en el aula. Las otras dos actividades que comentaremos se centran en dos tipos diferentes de textos: un dilema moral y un cuento infantil.

\section{«COMO ME VEO? ¿COMO ME VEN?: «EL ANIMAL AL QUE ME PAREZCO»}

La primera actividad que analizaremos es representativa del primer bloque de Aprender a vivir juntos en la escuela que trabaja el conocimiento de las personas, de uno mismo y de los demás. La actividad está diseñada para favorecer la expresión de la propia percepción y el grado en que los demás reconocen esa expresión como propia de una determinada persona. La secuencia metodológica que se propone a los profesores es la siguiente:

* En primer lugar el profesor propone a los niños que realicen, en un folio en blanco, un dibujo que represente «el animal al que se parecen» y les pide que escriban, detrás del folio, el porqué de su elección.

* Una vez terminadas las representaciones los niños colocan sus dibujos alrededor de la clase. A la vista de ellos, todos los niños deben intentar reconocer al menos cuatro de los dibujos y escribir los nombres de sus supuestos autores en un folio anexo, de manera que cada niño pueda comprobar hasta qué punto su dibujo ha sido reconocido por sus propios compañeros.

* Por último, la profesora modera una sesión en la que los niños emiten sus opiniones y realizan comparaciones en torno a los dibujos realizados. 
En la realización de esta actividad subyace la idea de que uno mismo puede expresarse a través de algún tipo de actividad externa y visible que le represente y a través de la cual puede ser reconocido por otros. La exposición de la propia imagen al reconocimiento por parte de los demás implica que la imagen con la que uno se representa a sí mismo puede resultar más o menos adecuada a la imagen que los demás le atribuyen. El ejercicio pretende alejar a los niños de una idea estática y esencialista de sí mismos y conducirlos a establecer una dialéctica entre la versión que producen de sí mismos y las que los demás le atribuyen. Se deriva de ello una idea interactiva y constructiva del propio yo, que lejos de abordarse como una entidad recóndita, difícilmente comunicable y cambiable, se plantea como un yo que se autopercibe, se expresa y es percibido por los demás; de un yo que se exterioriza y se reconstruye a partir de las propias representaciones y de las que los demás nos devuelven en una dialéctica entre el yo que me atribuyen y el que me atribuyo.

Con el fin de facilitar al profesor el establecimiento de un contexto que permita a los niños identificar la actividad como propia del área de educación socio-personal y diferenciarla de otras áreas educativas con las que puede confundirse, tales como la educación artística («dibujar»), o el conocimiento natural («conocer animales»), el proyecto propone a los profesores una introducción que permita reconocerla como una actividad de educación socio-personal y distinguirla de otro tipo de actividades que se realizan en otros contextos educativos como la que se cita a continuación:

"En la clase de boy vamos a bacer un ejercicio para conocernos mejor a nosotros mismos y para conocer a nuestros compañeros. Ese objetivo se puede conseguir de mucbas formas. Una manera de conseguirlo es bacer cosas a través de las cuales expresamos la manera en que nos vemos. Cuando nos vestimos, jugamos o dibujamos estamos manifestándonos. Esas cosas que bacemos les dice a los demás algo sobre nosotros, de manera que ellos pueden conocernos a través de nuestra forma de expresarnos. En la clase de boy cada niño va a dibujar el animal al que cree que se parece, y va a escribir, detrás del folio por qué razón ba elegido ese animals.

Si bien la intencionalidad de la actividad es clara en la propuesta, el significado que adquirirá para los niños en el aula se irá desvelando conforme se desarrolle el discurso comunicativo en el contexto social de la clase y previsiblemente a lo largo del mismo adquirirá matices distintos de los previstos en la propuesta hecha a los profesores. Las observaciones a las que nos referiremos a continuación corresponden al debate realizado en una clase de quinto curso y se refieren solamente a la tercera parte de la actividad que está planteada como un debate en el que participa toda la clase.

Aunque desde un punto de vista psicológico es clara la conexión proyectiva entre el dibujo y el autor, observamos que en la realización práctica de la actividad surgen problemas en el establecimiento de esa relación derivadas inicialmente de la forma en que la profesora maneja la situación didáctica. Los problemas arrancan del momento mismo en que inicia la sesión de debate grupal. Una vez què los niños han realizado sus dibujos y los han colocado en la pared, la profesora inicia el debate de esta forma:

Profesora: «Tendriamos que ver las características que tienen las personas que están aquí, analizar qué tipo de selva tenemos. Vamos a ver cada uno de los animales que aparecen y vais a ir diciendo características positivas y negativas de cada uno".

A continuación les propone que hablen de las características primero positivas y después negativas de los animales que se han representado y que ella va nombrando sucesivamente. Con el fin de garantizar un desarrollo ordenado de las intervenciones les pide a los niños que vayan levantando la mano cuando quieran intervenir. Ella va 
asignando turnos llamando a cada uno por su nombre, de manera que el discurso se va configurando como sigue:

Profesora: «Vamos a ver, primero el oso. Primero característicos positivas, Levantad la mano para intervenir. A ver, María.

Maria-Son fuertes.

P.TúJuan

Juan-Son muy ágiles para la pesca. (La profesora sigue nombrando a los niños por su nombre y asignándoles el turno de palabra).

Niñola-Son juguetones.

$P$ - ¿Veis alguna cualidad positiva más? (Silencio). Pues empezamos con las negativas.

$N$-Son muy gordos, les cuesta mucho trabajo correr.

$N$ - Son poco ágiles para cazar.

$N$-Son tontos, poco listos, porque cuando van a por miel, en vez de esperar a que las abejas se vayan... (Murmullos)...

P-Bueno, no conteis la película, ¿vale?. La pelicula la dejamos. Tú dices lo que te parece y se acaba. Y abora te callas un poquito, que otro niño quiere hablar (Murmullos). No hagais comentarios para ustedes nada más. Venga, algo más ¿no? (No bay más comentarios). Pasamos. Venga: el avestruz. Primero se Levanta la mano. Alejandro.

$N$ - Muy peligrosos.

$N$-Corre mucho.

$N$ - Es bonita.

$N$ - Es inteligente.

$P$ - Abora cualidades negativas.

$N-$ Yo no digo que es inteligente porque mete la cabeza en el boyo, porque metiéndola no consigue nada.

N-Es un poco fea.

Como puede observarse la profesora inserta en un fondo de regulación social de las intervenciones las observaciones acerca de los «animales». Utilizando la terminología de Bernstein, la profesora inserta constantemente las intervenciones que se refieren al contenido educativo socio-personal (discurso instruccional) dentro del marco que regula el funcionamiento social (discurso regulativo). Como telón de fondo, modera la dinámica social del grupo estableciendo normas en relación con la forma de intervención ( $P$ Primero se levanta la mano»), asignando turnos de palabra («A ver, María»; «Primero Juan»), garantizando condiciones de igualdad para todos los miembros, impidiendo que se monopolice la palabra ( Y ahora te callas un poquito que otro niño quiere hablar»), impidiendo que el diálogo se privatice («No hagais comentarios para ustedes nada más»), etc. El discurso instruccional referente al contenido propiamente socio-personal aparece en las intervenciones en las que la profesora establece la secuencia de las intervenciones ( Primero características positivas»...»Empezamos con las negativas») así como el criterio de lo que acepta como intervenciones válidas o no para el contexto de educación socio-personal. Vemos que la profesora acepta un tipo de intervenciones como las que califican las cualidades de los animales y desautoriza otras que estima no adecuadas al contexto, como la intervención que intenta extenderse abundando en el comportamiento obstinado y apresurado de los osos. En este caso, el niño está introduciendo una imagen cinematográfica en la reflexión sobre los animales que representan a los niños, pero la profesora estima que ese tipo de conocimiento es extra-escolar, procede del cine y, en consecuencia, no cabe en la situación actual. La profesora está exhibiendo así explícitamente un criterio que permite a los niños el reconocimiento de cuáles son las intervenciones que son y que no son pertinentes al contenido que se discute.

El hecho de que la profesora deba realizar este doble discurso de manera simultánea y jerarquizada canaliza la comunicación en el aula en una dirección mientras que obstruye otras. El control de las intervenciones de manera que todos los niños participen en la discusión y la preocupación porque el comentario abarque las características de todos los animales en un orden que ella establece ( $($ Primero positivas y después negativas») le permite un tratamiento en extensión del tema, que asegura un 
repaso superficial de las cualidades de los animales pero cierra el paso a intervenciones que podrían significar una más profunda exploración de los representaciones hechas por los niños. La necesidad de regular el orden social de la clase y el criterio de comentar todos los animales tiene el riesgo de que el debate se convierta en un ordenado pero anodino repaso sobre las características de los animales que poco tienen que ver con el conocimiento de las personas. Como señalábamos anteriormente, la actividad se desarrolla, desde la presentación del debate inicial por parte de la profesora, dentro de una ambigüedad que se desliza entre el objetivo de conocer a las personas («las características de las personas que están aquí») y la representación elegida como forma de expresión de esas personas («analizar qué tipo de selva tenemos»). En esta presentación la profesora identificó ambos aspectos de la actividad y el desarrollo del discurso no nos permite reconocer si la conexión entre las representaciones (los dibujos) y sus significados (las características de los niños) queda clara para los niños. Sin embargo, si el debate sobre los «animales» no les permite establecer una conexión entre los autores y sus representaciones, entonces la reflexión sobre uno mismo y el enriquecimiento de la propia representación puede verse sustituído por una mera enumeración acerca de las caracteríticas de figuras que se han alienado de sus autores y se han convertido en una selva de animales yuxtapuestos que poco tienen que ver con aumentar el conocimiento del grupo de niños que los han producido.

\section{CUENTO Y DILEMA MORAL}

Nos referiremos a continuación a dos actividades en las que un texto externo mediatiza la actividad educativa en el ámbito de la educación moral. Se trata de un dilema moral kohlberiano y de una pequeña historia titulada «El club», incluída dentro del libro Saltamontes va de viaje del escritor de cuentos infantiles Arnold Lobel (1978). Uno y otro texto son portadores en sí mismos de ideas acerca del comportamiento moral y acerca del funcionamiento social. Sin embargo se trata de dos diferentes tipos de textos tanto desde un punto de vista formal como desde la perspectiva de los significados que vehiculizan, las actividades que posibilitan y los tipos de discursos morales que generan. Mientras que el dilema es un texto construído explícitamente para suscitar el razonamiento moral, el cuento sólo lo es indirectamente. Básicamente es un texto literario no diseñado en principio para ser utilizado como un texto de educación moral. Los niños pueden leerlo en situaciones no escolares como actividad de ocio, o bien en la escuela en las clases de lengua. En el contexto de la educación socio-personal y moral, la lectura del cuento se realiza con una intención educativa en la que al interés literario del texto se une la finalidad de promover una serie de objetivos propios del ámbito.

\section{Un dilema moral en torno a lo que significa ser miembro de un jurado en la escuela}

La actividad en la que se discute el dilema moral se propone en el proyecto Aprender a vivir juntos en la escuela en el bloque sobre las «relaciones interpersonales» y pretende trabajar los conflictos que surgen entre las relaciones de amistad y la obligación moral. El texto del dilema es éste:

María es una niña de segundo curso. En su escuela se ba organizado un concurso de disfraces para el carnaval. Las reglas del concurso dicen que se elegirán los disfraces más originales que bayan diseñado los propios niños. Maria es miembro del jurado y tiene que dar su opinión según las reglas del concurso. Pedro y Laura, que son los mejores amigos de Maria se presentan al concurso. La verdad es que sus trajes no son muy originales y además, ellos no ban diseñado sus trajes. Si María les da una baja puntuación, sabe que 
Pedro y Laura se enfadarán y que quizás dejen de hablarle. ¿Cuál creeis que debería ser la opinión de María como miembro del jurado?».

En este caso, la secuencia para el desarrollo pedagógico de la actividad que se propone en el Proyecto es la siguiente:

* En primer lugar el profesor lee el dilema a los niños.

* A continuación se establece una discusión en pequeños grupos en torno a algunas preguntas planteadas por el profesor como las siguientes:

- ¿Qué creeis que debería bacer Maria?.

- ¿Creeis que Pedro y Laura se enfadarian si María les diera una baja puntuación?

- ¿Debería Maria darles una puntuación alta con tal de que no se enfadaran con ella?.

- ¿Quépodría suceder si María les diera una buena puntuación?.

- ¿Quépodria suceder si Maria les diese una baja puntuación?.

* Una vez finalizadas las discusiones, un miembro de cada grupo expone los argumentos que se ban defendido en su equipo al conjunto de la clase. Se ponen en comín las conclusiones de los grupos y se introducen nuevas cuestiones que dirigen la atención de los niños a reflexionar en otras situaciomes similares vividas o conocidas por ellos mismos, tales comoéstas:

- ¿Habeis vivido alguna vez alguna situación parecida a la de esta bistoria?. Contar lo que sucedió.

- ¿Quéopinais de la forma en que se resolvió?.

- ¿Podría hacer habido otras soluciones mejores?

El contenido del dilema moral habla de una situación social en que las personas realizan distintos tipos de actividades que pueden entrar en conflicto. Por ejemplo las personas tenemos vínculos con nuestros amigos y a veces tenemos que desempeñar papeles sociales, como ser miembro de un jurado, en las que tenemos que comportarnos de acuerdo con ciertas reglas, cuyo seguimiento puede acarrearnos la pérdida de nuestros amigos. En situaciones como éstas debemos decidir entre preservar a nuestros amigos o comportarnos de acuerdo con ciertas reglas que garantizan un tratamiento en condiciones de igualdad a todas las personas que participan en ellas.

\section{Una historia de grupos sectarios y viajeros solitarios}

En el segundo texto se narran las aventuras de un personaje viajero e independiente que se va encontrando con gentes que se aferran rígidamente a posturas y creencias. El argumento de la historia es el siguiente:

En algún lugar de su viaje en solitario, Saltamontes encuentra a un numeroso grupo de escarabajos que adoran la mañana y que expresan su gusto común con carteles alusivos a que no bay otro momento del día comparable a ese. Son miembros del "Club de los amantes de la mañana" que admiten a Saltamontes basta que descubren que también a éste le gustan otros momentos del día como la tarde y la noche. En ese momento los escarabajos le insultan y le expulsan del club. Saltamontes sigue su camino.

Como el dilema moral, el cuento es también portador de una idea del mundo social, de las relaciones y de los conflictos entre las personas y de posturas y formas de solución. El cuento vehiculiza la idea de que algunos grupos se aferran sectaria y acríticamente a ciertas cuestiones, mientras que algunos individuos sostienen una posición individual: hacen un camino en solitario sin aceptar las presiones de los grupos corriendo el riesgo de quedar separados. La intención educativa de esta actividad es suscitar la reflexión en torno a estas dos posturas.

La secuencia de desarrollo que se propone en el Proyecto para el desarrollo de esta actividad es en parte similar y en parte diferente de la anterior:

* El profesor lee el cuento a los niños.

* A continuación se suscita un diálogo colectivo (en gran grupo o en grupo restringidos) en el que se plantean dos tipos de cuestiones: relacionadas con el propio cuento unas y otras dirigidas a la personalización de la problemática planteada en él. Entre las primeras están: 
- ¿Qué defendían los escarabajos del club?.

- ¿Cómo recibieron a Saltamontes los miembros del Club de la mañana?.

- ¿Por qué le insultaron y le echaron del club?

- ¿Qué pensais sobre su comportamiento?.

- ¿Qué pensais sobre el comportamiento de Saltamontes?.

- ¿Hizo bien Saltamontes en manifestar lo que le gustaba?.

- ¿Debería haberlo hecho si hubiera sabido que le iban a echar del club?

Ejemplos del segundo tipo de preguntas son las siguientes:

- ¿Habeis vivido alguna situación parecida a la que se narra en el cuento?.

- ¿Qué sucedió?.

- ¿Fuisteis excluídos?

- ¿Fuisteis vosotros los que excluisteis a alguien?. ¿Por qué razón?.

* Por último el profesor pide a los niños que reelaboren el cuento de manera que cada uno encuentre la solución que le parezca más conveniente.

\section{Diferentes medios, diferentes mensajes}

El dilema moral suscita un número relativamente pequeño de respuestas por parte de los niños. De hecho caben solamente dos comportamientos posibles ante el dilema: o inclinarse por la opción favorable a los amigos o adoptar una posición favorable al seguimiento de las normas del concurso. Yendo más lejos todavía, puede decirse que el dilema moral tiene garantizada de entrada la opción por la que los niños deben inclinarse. Supuesto que «María debe dar su opinión según las reglas del concurso», la pregunta acerca del comportamiento que la protagonista debe tener puede considerarse como una seudopregunta en el sentido de que la respuesta está dada por adelantado en el propio enunciado formal del dilema. La respuesta está determinada también por el propio contexto social en el que el dilema se desarrolla: se trata de un concurso escolar en el que María es juez, de manera que su decisión no es una cuestión meramente personal o interpersonal, sino que tiene una dimensión institucional en cuanto que es responsable ante la propia escuela que organiza el concurso y su actuación será objeto de aprobación o desaprobación pública. Y de la misma manera que María debe tomar su decisión en un contexto institucional, los niños deben dar una respuesta al dilema en un contexto institucional en el que el profesor detenta una posición de autoridad y espera que los niños se adapten a las reglas del juego escolar. En este contexto es altamente improbable que los niños se inclinen por la alternativa de no seguir las reglas del concurso.

Las observaciones realizadas en segundo y quinto curso de primaria confirmaron que los niños de ambos niveles se inclinaron claramente por la solución de seguir las reglas en un clima caracterizado por intervenciones mínimas de la profesora que se limitaba a controlar el orden de intervención de los niños.

Veamos un ejemplo de la discusión en un aula de segundo curso:

Profesora- "¿Cuál creeis que debe ser la opinión de María como miembro del jurado?.

Niñola-Si no se lo ban inventado ellos que le ponga algo bajito.

$N$ - Que ponga una nota baja porque tienen que seguir las reglas del concurso.

$P-i$ Creeis que Pedro y Laura se enfadarian si le diesen una puntuación baja?

$N$-Si eran comprensivos no se enfadarian con ella y al cabo de un tiempo, si se enfadan, se bablarian con ella.

$N$ - Si son buenos amigos, no se enfadarian.

$N$ - No se deberian enfadar porque si bacian otro concurso y se invantaban los disfraces, podrian ganar».

En quinto curso los niños abundaron también en la idea de que lo correcto era seguir las reglas, aunque introdujeron algunos matices que conviene observar:

Profesora- ¿Cuál creeis que debe ser la opinión de Maria como miembro del jurado?.

Niñola - Les daria la nota que se merecen y si se enfadan les diría que soy del jurado y tengo que cumplir las reglas aunque sea su amigo. No me dejaría influir a pesar de que supiera que se iban a enfadar mis amigos. 
$N$ - Si se enfadaban por eso es porque son amigos de conveniencia y no amigos de verdad. Yo no me enfadaria. $N$ - Les pondría la puntuación que les corresponde y después del concurso intentaría ser su amigo de nuevo, pero si ellos no quieren, me buscaría amigos nuevos.

$N$ - Yo buscaría a otro para el jurado. Yo me iría y que fuera otro el que diera los puntos. Asi no influiria en mis amigos y evitaría el enfadarme con ellos».

Parece pues que la clave educativa del dilema moral no está en la respuesta a la pregunta: «¿Qué debe hacer María como miembro del jurado?», sino en el tipo de argumentos justificativos que esa pregunta suscita, argumentos que configuran una determinada representación del ámbito moral que implica una distinción entre diferentes aspectos de uno mismo: el yo público y el privado («soy miembro del jurado y tengo que cumplir las reglas aunque sea su amigo»), el yo que tiene responsabilidades institucionales y el yo de las relaciones interpersonales. Esos dos aspectos del yo aparecen jerarquizados, de manera que el yo público, el yo del deber moral está por encima del yo amistoso, y de esa jerarquización se deriva también una diferenciación entre dos tipos de amistad: «los amigos de verdad" y «los amigos de conveniencia» que también están jerarquizadas, de manera que sólo los amigos comprensivos, los que entienden la prioridad del comportamiento justo merecen ser mis amigos. En caso contrario uno debe abandonarlos y buscar otros. Porque la opción de conservar los amigos que no se atienen a las reglas establecidas aparece como incompatible con la adopción de una decisión justa. Y si aquella es la opción, entonces lo oportuno es abandonar el jurado.

Como en el dilema moral, las posturas que se enfrentan en el cuento tienen que ver con el mantenimiento de la independencia personal frente a las relaciones que nos vinculan con otros (los amigos, el grupo). Ambos textos tienen sus propios mecanismos para inclinar al niño a favor de las posturas que representan sus protagonistas, aunque, los protagonistas de ambos textos tienen motivos diferentes para comportarse como lo hacen: Saltamontes no tiene ninguna constricción externa para ser un viajero solitario; es su opción personal. María sin embargo tiene un deber que cumplir: tiene una responsabilidad social y debe atenerse a unas normas obligatorias.

Sin embargo, a pesar de que la historia es claramente favorable a la opción individual del protagonista, el texto no suscita en los niños posturas incondicionalmente favorables a ella. En realidad las interpretaciones de los niños van más allá del planteamiento del propio autor de la narración. El cuento no les pide a los niños que decidan acerca de la mejor solución, y más allá de las intenciones del autor, despierta en los niños la exploración de los motivos de las partes implicadas. Y esa comprensión de las perspectivas en conflicto es filtrada por la forma en que el conflicto resuena en los intérpretes del cuento, de manera que las lecturas que el texto genera son tan diversas como las posiciones de los lectores. De esta posibilidad de generar lecturas divergentes se deriva también la de reescribirlo. En la última parte de la actividad se les pide a los niños que reconstruyan el cuento encontrando finales diferentes y más adecuados a las soluciones que a ellos les gustaría que se produjeran. Esta última parte nos permite comprobar las posibilidades educativas que ofrece el cuento como modalidad de educación socio-personal y moral, posibilidades que se derivan de ofrecer al niño la oportunidad de convertirse en autor de un nuevo cuento en el que los personajes serán vistos desde ángulos nuevos y las relaciones y los conflictos entre ellos darán lugar a nuevas articulaciones del problema no previstas en la versión original.

En el cuadro que presentamos a continuación (Tabla I) aparecen algunos algunos de los finales reinventados por los niños de quinto curso y sus respuestas a nuestra pregunta sobre cuál podría ser la enseñanza de su propio cuento: 
TABLA I

\begin{tabular}{ll}
\hline TEXTO REINVENTADO & RESPUESTA A LA PREGUNTA: «Si tú \\
\hline & fueras la profesora, cपué crees que podríar \\
& aprender los niños con tu cuento?»
\end{tabular}

Enrique:

«El saltamontes se fue muy enfadado, después de haberle echado del «Club de la mañana». Empezó a caminar y caminar hasta que se encontró a unas hormigas cazadoras que empezaron a perseguirle. Las hormigas le acorralaron y de repente salió una araña y enredó a las hormigas una a una y se las fue comiendo. El saltamontes salió corriendo, pero al ir saltando no se dio cuenta de que se había metido en un nido de arañas».

\section{Enrique:}

«Que es difícil elegir, porque, si estás solo y quieres estar con alguien, a lo mejor dejas a unos amigos y no sabes si luego vas a encontrar a otros».
Luis:

«El saltamontes cuando se enteró que la regla era que tenía que gustarle la mañana se fue. Los escarabajos se quedaron hablando en voz baja y se dieron cuenta de que le tenía que gustar también la tarde y la noche. Después de muchos años el saltamontes volvió y encontró que el «club de la mañana»se llamaba el «club del día» y los escarabajos se disculparon por haber sido tan cabezotas».
Luis:

«Que si alguien te propone algo, tú no tienes que decirle que no. Tienes que pensarlo mejor y si te parece bien, puedes aprender de éllo que tú no sabes».

\section{Rocío:}

«A mí también me gusta mucho la tardedijo el saltamontes-.

- ¿Qué?, dijo el escarabajo.

- Y la noche, dijo otra vez.

- ¡Fuera de aquí! dijeron todos los escarabajos

El Saltamontes les explicó que la noche era un momento donde iba a empezar la mañana y que la tarde se convertía en noche para empezar luego la mañana. Todo se arregló y el Saltamontes siguió con los escarabajos».

\section{Rocío:}

«Pues que los escarabajos no comprendían lo que Saltamontes le estaba diciendo, no querían comprender que a él le gustaban además otras cosas, y que hay que intentar hacer comprender a los demás cuando se hacen cosas nuevas».

\section{Sergio:}

«El saltamontes se fue muy enfadado. Entonces decidió hacer un club de la noche y buscó a mucha gente. Pero un día un escarabajo de club de la mañana pasó al lado del club de la noche y fué rápidamente a su club. Entonces estalló una guerra entre saltamontes y escarabajos. Dos semanas después estaban todos heridos. Entonces los dos jefes decidieron juntarse en un sólo club: el «club de todo el día».

\section{Sergio:}

«Que a veces es mejor unirse que declararse la guerra y que haya heridos por todas partes".

A nuestro entender estas respuestas muestran la capacidad del texto narrativo para generar reescrituras divergentes. Mientras que Enrique enfatiza las dificultades que puede encontarse el protagonista que decide separarse del grupo y continuar su camino en solitario, Luis introduce una capacidad de reflexión en el grupo de escarabajos de la que fueron privados por el autor original del cuento. Las opciones de Rocío y Sergio son conciliadoras: los personajes enfrentados llegan finalmente a con- 
consigue flexibilizar la postura sectaria del grupo de escarabajos, mientras que en el caso de Sergio, la solución conciliadora aparece tras experimentar las consecuencias desastrosas a las que conduce el enfrentamiento.

La observación del discurso generado en el aula permite confirmar la idea inicial de que dilema y cuento proponen a los niños dos ideas diferentes aunque complementarias del mundo social: mientras que el dilema les plantea la idea de un mundo justo organizado según reglas de validez universal, la narrativa les introduce en la aventura de explorar situaciones concretas en las que se suscitan conflictos entre personas que tienen diferentes perspectivas y diferentes motivos para comportarse como lo hacen. Y en la medida en que los niños pueden acceder a través de ella a la comprensión simultánea de las perspectivas en conflicto, las narrativas les introducen en el sentido de una cultura que puede ser negociada y reconstruída entre sus miembros.

\section{Referencias}

APPEL, M. W. (1979). Ideología y curriculo Madrid. Akal.

BENHABIB, S. (1992). Una revisión del debate sobre las mujeres y la teoría moral. Isegoria, 6, 37-63.

BERNSTEIN, B. (1977). Class, codes and control. Londres: Routledge Kagan. (Trad. cast.: Clases, códigos y control.Vol. I: Estudios teóricos para una sociología del lenguaje. Vol ll: Hacia una teoría de las transmisiones educativas. Madrid. Akal.)

BERNSTEIN, B. (1990a). The Structuring of Pedagogic Discourse. Volume IV. Class, codes an control. Londres. Routledge. (Trad. cast.: La estructura del discurso pedagógico. Madrid. Morata 1993.

BERNSTEIN, B. (1990b). Poder, educación y conciencia. Barcelona. El Roure.

BORREGO DE DIOS, C. (1991). El papel de las narrativas en el conocimiento social. Investigación en la escuela, 15, 16-29.

BORREGO DE DiOS, C. (Ed.). (1992a). Curriculum y desarrollo socio-personal. Sevilla. Alfar.

BORREGO DE DIOS, C. (1992b). El desarrollo socio-personal como ámbito específico de contenidos escolares: una propuesta educativa. En Borrego de Dios (Ed.) (1992a).

BORREGO DE DIOS, C. (Coord). (1992c). Aprender a vivir juntos en la escuela. Sevilla. Junta de Andalucía.

Bruner, J. (1986). Actual Minds, Possible Worlds. Harvard University Press, Cambridge, Mass. (Trad. cast.: Realidad mental y mundos posibles. Barcelona. Gedisa.)

Bruner, J. (1990). Acts of meaning. Cambridge, Mass. Harvard Universitu Press. (Trad. cast.: Actos de significado. Madrid. Alianza. 1992.)

BUXARRAIS, M. R., y otros. (1990). Etica i escola: el tractament pedagògic de la diferència. Barcelona. Rosa Sensat Edicions 62.

CARR, W., y KEMMIS. (1986). Becoming critical. (Trad. cast.: Teoria crítica de la enseñanza. La imvestigaciónacción en la formación del profesorado. Barcelona. Martínez Roca.)

DeCreto de EdUCACIÓN PRIMARIA (1992). Sevilla. Junta de Andalucía.

Diseños CuRRICULARES DE LA REFORMA. (1990). Areas y ámbitos de conocimiento y experiencia. Sevilla. Junta de Andalucía.

EDWARDS, D., y MERCER, N. (1987). Common knowledge. The development of understanding in the classroom. (Trad. cast.: El conocimiento compartido. El desarrollo de la comprensión en el aula. Barcelona, Madrid. Paidos/MEC.)

EllenWOOD, S., y RYAN, K. (1991). Literature and Morality: An Experimental Curriculum. En Kurtiness y Gewirtz: Handbook of Moral Bebaviour and Development. Vol 3: Application. Hillsdale, Nueva Jersey. L.E.A.

ELLIOTT, J. (1990). La investigación-acción en educación. Madrid. Morata.

ESPINOLA, V. (En prensa). Principios y prácticas de adecuación curricular. En: Temps $d$ Educació. Universidad de Barcelona.

GILLIGAN, C. (1982). In a different voice. (Trad. cast.: La moral y la psicología del desarrollo femenino. México. FCE.)

Gilligan, C.; Ward, J. V., y Taylor, J. M. (1988). Mapping the Moral Domain. Cambridge, Mass. Harvard University Press.

Hersh, R,; Reimer, J., y PAOLITO, D. (1979). Promoting Moral Growth from Piaget to Koblberg. Nueva York: Longman. (Trad. cast: El crecimiento moral de Piaget a Koblberg. Madrid. Narcea.)

INGHILLRI, M. Talk, interaction and the organization of knowledge in the science classroom». En prensa en: Temps $d$ Educació. Universidad de Barcelona.

JORDAN, J. A., y SANTOLARIA, F. F. (Eds). (1991). La educacion moral boy. Cuestiones y perspectivas. Barcelona. 1987. PPU. 
KOHLBERG, L. (1966). A cognitive development analysis of children's sex role concepts and attitudes. En E. E. Maccoby (Ed.): The Development of Sex Differences. (Trad. cast.: Desarrollo de las diferencias sexuales. Madrid. Morata.)

KOHLBerg, L. (1968). The child as a moral philosopher. Psychology Today. (Trad. cast. en J. Delval (Ed.): Lecturas de psicología del niño. Vol. 2. Madrid. Alianza Universidad.)

KOHLBERG, L. (1969). Stage and sequence: The cognitive-developmental approach to socialization. En A. Goslin (Ed.). Handbook of socialization theory and research. Chicago. Rand McNally.

Kohlberg, L. (1971). From is to ought. En Mischel, T. (Ed.): Cognitive Development and Epistemology. Nueva York. Academic Press.

KoHLBerg, L. (1975). The Cognitive Developmental Approach to Moral Educcation. Thecletta Kappla, 56, 10, 670-677. (Trad. cast.: El enfoque cognitivo-evolutivo de la educación moral. En Jordan y Santolaría, 1991.)

LArrosa, J. (1993). The Pedagogic Construction of the Moral Domain and Moral Subject: an exploratory description of three pedagogic practices in moral education. Curriculum Studies, $1,1$.

LOBEL, A. (1978). Grasshopper on the road. (Trad. cast.: Saltamontes va de viaje. Madrid. Alfaguara, 1991.)

MarTinez, M., y PUIG, J. M. (Coord. 1991). La educación moral. Barcelona. Graó, ICE Universidad de Barcelona.

Mead, G. H. (1934). Mind, Self and Society. Chicago. The University Chicago Press. (Trad. cast.: Espíritu, persona y sociedad. Buenos Aires. Paidós, 1990.)

Perez-Delgado, E., y Garcia-Ros, R. (1991). La psicología del desarrollo moral. Madrid. Siglo XXI.

PIAGET, J. (1932). Le jugement moral chez l'enfant. París. Presses Universitaires de France. (Trad. cast.: El criterio moral en el niño. Barcelona. Fontanella.)

PUIG, J., y MARTINEZ, M. (1989). Educación moral y demacracia. Barcelona. Laertes.

SELMAN, R. L. (1980). The Growth of Interpersonal Understanding: Developmental and Clinical Analysis. Nueva York. Academic Press.

SADOVNIK, A, R. (1991). La teoría de la práctica pedagógica de Basil Bernstein: Un enfoque estructuralista. Investigación en la escuela, 1992, 17, 7-31.

SELMAN, J. (1980). Concepts of self and morality: Women's reasoning about abortion. Nueva York. Praeger.

STUBBS, M. (1983). The Sociolinguistic Analisis of Natural Language. En Blackewell Pudliher Litd. (Trad. cast.: Análisis del discurso. Madrid. Alianza.

TURIEL, E. (1978). Distinct conceptual and developmental domains: Social convention and morality. En C. B. Keasy (Ed.): Nebraska Symposium on Motivation. Social Cognitive Development. Vol 25. Lincoln, N.B. University of Nebraska Press.

TURiEi, E.; ENeSCO, I., y LiNAZA, J. (Comps). (1989). El mundo social en la mente de los niños. Madrid. Alianza. 


\section{Una aproximación teórico crítica al análisis} de la práctica educativa sobre educación moral Concepción Borrego de Dios CL\&E, 1994, 21, pp. 53-66

Resumen: El artículo realiza un análisis crítico en torno a algunas actividades incluídas en el proyecto Aprender a vivir juntos en la escuela (Borrego de Dios (Coord. 1992c), diseñado para facilitar la práctica educativa en educación socio-personal y moral en aulas de educación primaria en el contexto de la Reforma Educativa en la Comunidad Autónoma Andaluza. A través del análisis de tres actividades educativas (una actividad de autoexpresión, un dilema moral y un cuento infantil) y de la observación etnográfica de su puesta en práctica en aulas de segundo y quinto curso de educación primaria, el artículo focaliza dos tipos de cuestiones teórico-prácticas relacionadas con la educación moral. La primera contempla el problema del desarrollo de un diseño curricular de educación socio-personal en la práctica educativa del aula desde la perspectiva teórica de B. Bernstein (1990a y 1990b), quien propone que el discurso pedagógico implica un proceso de recontextualización en el que un discurso instruccional se inserta dentro del dominante discurso regulativo. La segunda cuestión enlaza con la existencia de dos diferentes planteamientos en torno a la educación moral: la tradición cognitivo-evolutiva que tiene su máxima expresión en los planteamientos educativos kohlberianos $(1969,1971,1976)$ y los recientes enfoques que parten de una concepción interpretativista del mundo social, de los que se desprende una posición narrativa en relación con el ámbito socio-personal y moral (Bruner 1986, 1990; Gilligan, 1982).

Datos sobre la autora: Actualmente la autora trabaja en el diseño y desarrollo curricular en el ámbito del desarrollo socio-personal y moral desde una perspectiva socio-constructivista. Recientemente está ampliando su campo de interés al papel de la Televisión como mediador semiótico en la construcción del mundo social

Dirección: Dpto. de Psicología Evolutiva y de la Educación. Universidad de Sevilla. Escuela de Magisterio. Avda. Ciudad Jardín, s/n. 41005 Sevilla.

(C) PERMISOS PARA CITAR O REPRODUCIR EN OTRAS FUENTES: Se pueden citar libremente hasta 500 palabras. Para reproducir una porción de texto mayor, figuras o ilustraciones, se deberá pedir permiso por escrito a la revista, especificando el uso al que se destina el texto. En todos los casos, se deberá citar el copyright de CL\&E. En el caso de artículos o textos que hayan sido a su vez reproducidos en $C L \& E$ los interesados deberán dirigirse tanto a los detentadores del copyright original como a $C L \& E$, en el caso de que se quiera hacer uso de la traducción. FOTOCOPIAS: Para todo lo relacionado con el uso mediante fotocopia del material de esta revista, deberán dirigirse a: CEDRO, C/ José Marañón, 10, $3 .^{\circ}$ Izda. Tel. 5941575 . Fax 4453567

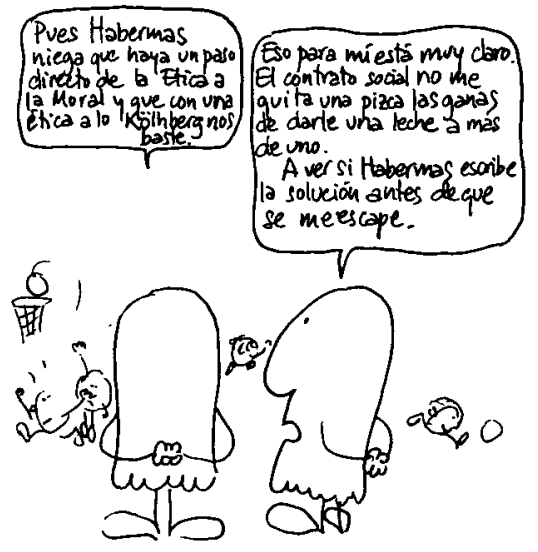

